\title{
3D FACE IDENTIFICATION BASED ON ARRANGEMENT OF SALIENT WRINKLES
}

\author{
G. Antini, S. Berretti, A. Del Bimbo and P. Pala \\ Dipartimento di Sistemi e Informatica \\ University of Firenze, Firenze, Italy
}

\begin{abstract}
In this paper, we propose an original framework for three dimensional face representation and matching for identification purposes. Basic traits of a face are encoded by extracting curves of salient ridges and ravines from the surface of a dense mesh. A compact graph representation is then extracted from these curves through an original modeling technique capable to quantitatively measure spatial relationships between curves in a three dimensional space. In this way, face recognition is obtained by matching $3 \mathrm{D}$ graph representations of faces. Experimental results on a 3D face database show that the proposed solution attains high recognition accuracy and is quite robust to facial expression and pose changes.
\end{abstract}

\section{INTRODUCTION}

In the last years, several works have addressed the issue of face identification mainly focussing on detection and recognition of faces in still images and videos (see [1] for an updated survey). More recently, the increasing availability of three-dimensional (3D) data, has paved the way to the use of 3D face models to improve the effectiveness of face recognition systems [2]. In fact, solutions based on 3D face models, feature less sensitivity - if not invariance - to lighting conditions and pose. This is particularly relevant in real contexts of use, where face images are usually captured in non-controlled environments, without any particular cooperation by human subjects.

Generally, three main classes of approaches can be identified to distinguish the way in which $3 \mathrm{D}$ face models can improve face identification with respect to traditional solutions. A first class of approaches relies on a generic 3D face model to match two 2D face images. For example, in [3] a method is proposed for face recognition across variations in pose, ranging from frontal to profile views, and across a wide range of illuminations, including cast shadows and specular reflections. To account for these variations, the algorithm simulates the process of image formation in 3D space, using computer graphics, and it estimates 3D shape and texture of faces from single images.

A different class of approaches relies on using multiple imaging modalities in which information extracted from 3D shapes and 2D images of the face are combined together to attain better recognition results. In [4], face recognition in videos is obtained under variations in pose and lighting by using $3 \mathrm{D}$ face models. In this approach, 3D database models are used to capture a set of projection images taken from different point of views. Similarity between a target image and $3 \mathrm{D}$ models is computed by matching the query with the projection images of the models. In [5], Gabor filter responses in the $2 \mathrm{D}$ domain, and "point signature" in the $3 \mathrm{D}$ are used to perform

This work is partially supported by the Information Society Technologies (IST) Program of the European Commission as part of the DELOS Network of Excellence on Digital Libraries (Contract G038-507618). face recognition. Extracted 2D and 3D features are then combined together to form an augmented vector which is used to represent each facial image. PCA-based recognition experiments, performed using $3 \mathrm{D}$ and 2D images are reported in [6]. The multi-modal result was obtained using a weighted sum of the distances from the individual $3 \mathrm{D}$ and 2D face spaces. A large experimentation in terms of number of subjects, gallery and probe images, and the time lapse between gallery and probe image acquisition, is also presented in this work.

Finally, another class of methods relies on using only 3D shapes for the purpose of face recognition. Early works focused on the use of surface curvature information and the Extended Gaussian Image, which provide one-to-one mapping between curvature normals of the surface and the unit sphere. Following a similar solution, 3D face recognition is approached in [7], by first segmenting the shape based on Gaussian curvature, and then creating a feature vector from the segmented regions. This set of features is then used to represent faces in recognition experiments. However, a key limitation of such approaches is that to enable reliable extraction of curvature data, accurate $3 \mathrm{D}$ acquisition is required. Other solutions have used registration techniques to align 3D models or clouds of points. In [8], face recognition is performed using Iterative Closest Point (ICP) matching of face surfaces with resolution levels typical of the irregular point cloud representations provided by structured light scanning.

In this paper, we propose an original solution to $3 \mathrm{D}$ face identification based on the extraction, representation and matching of salient creases of 3D faces. This model is mainly motivated by psychological studies, indicating that a set of simple lines characterizing the structure of an object are sufficient to identify its shape [9]. In fact, line segments are less sensitive to illumination changes and local variations since they integrate the inherent local structural characteristics with spatial information of a face. In our approach, 3D face models are represented by salient ridges and ravines, that we generally call wrinkles, extracted by using curvature information on the surface of a dense mesh. Facial information captured by these curves is then represented in compact form evaluating spatial relationships between every pairs of curves. To this end, we propose an original modeling technique capable to quantitatively measure the spatial relationships between three dimensional entities. The model develops on the theory of weighted walkthroughs (WWs), originally proposed to represent spatial relationships between two-dimensional extended entities [10]. In particular, the discussion starts by focusing on volumetric entities, then it is shown that the model can also capture relationships between linear entities. Finally, mapping wrinkles and their relationships to a graph model and defining a distance measure between 3DWWs allows for the effective comparison of face models

The paper is organized in three Sections and a Conclusion. In Sect.2, a method is presented for extracting salient curves of ridges and ravines from a dense triangular mesh. The theory of 3DWWs is then developed and proposed to represent spatial relationships between linear entities in a 3D space. This enables the effective rep- 
resentation of a face model through an attributed relational graph accounting for salient face wrinkles and their relationships. Based on this model, a method for the efficient comparison of graph representations of facial models is discussed in Sect.3. Face recognition results obtained on a 3D face database are reported in Sect.4. Finally, conclusions are outlined in Sect.5.

\section{EXTRACTION AND DESCRIPTION OF WRINKLES}

Surface creases, ravines and ridges, provide important information about the shape of 3D objects. Intuitively, these salient traits can be defined as those curves on a surface where the surface bends sharply. Loci of sharp variation of surface normals have been intensively studied in connection with researches on surface mathematics, human perception of shapes, quality-control of free-form surfaces, image and data analysis and many other applications. In the proposed solution, computation of salient convex creases (ridges) and concave creases (ravines) is accomplished through the technique described in [11]. As an example, Fig.1 shows salient curvature triangles detected on a face model. Triangles in Fig.1(a) show model ridges, while ravines are highlighted by the triangles in Fig.1(b).

Wrinkles extracted from a 3D face are 1D curves in a 3D reference space. Information captured by these curves is represented by modeling curves and their mutual spatial relationships. To this end, we propose a theory of 3D spatial relationships between linear entities, which develops on the model of weighted walkthroughs (WWs) originally defined for two-dimensional extended entities [10]. Description of spatial relationships through 3DWWs is invariant to translation and scaling but not to rotation. Therefore, in order to enable invariance of face matching with respect to translation, scaling and rotation, face models are first normalized: models are scaled and rotated so as to fit within a sphere of unit radius centered at the nose tip and aligning the nose ridge along the $Z$ axis.
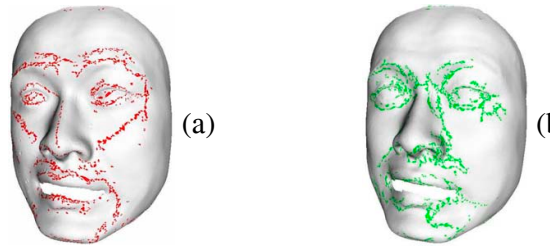

Fig. 1. Salient curvature extrema detected on a face model: triangles of ridge (a) and ravine (b).

\subsection{D Weighted Walkthroughs}

In a three dimensional Cartesian reference system, with coordinate axes $X, Y, Z$, projections of two points, $a=\left\langle x_{a}, y_{a}, z_{a}\right\rangle$ and $b=$ $\left\langle x_{b}, y_{b}, z_{b}\right\rangle$ on each axis, can take three different orders: before, coincident, or after. The combination of the three projections results in 27 different three-dimensional displacements (primitive directions), which can be encoded by a triple of indexes $\langle i, j, k\rangle$ :

$i=\left\{\begin{array}{cc}-1 & x_{b}<x_{a} \\ 0 & x_{b}=x_{a} \\ +1 & x_{b}>x_{a}\end{array} \quad j=\left\{\begin{array}{cc}-1 & y_{b}<y_{a} \\ 0 & y_{b}=y_{a} \\ +1 & y_{b}>y_{a}\end{array} \quad k=\left\{\begin{array}{cc}-1 & z_{b}<z_{a} \\ 0 & z_{b}=z_{a} \\ +1 & z_{b}>z_{a}\end{array}\right.\right.\right.$

In general, pairs of points in two sets $A$ and $B$, can be connected through multiple different primitive directions. According to this, the triple $\langle i, j, k\rangle$, is a walkthrough from $A$ to $B$ if it encodes the displacement between at least one pair of points belonging to $A$ and
$B$, respectively. In order to account for its perceptual relevance, each walkthrough $\langle i, j, k\rangle$ is associated with a weight $w_{i, j, k}(A, B)$ measuring the number of pairs of points belonging to $A$ and $B$, whose displacement is captured by the direction $\langle i, j, k\rangle$.

The weight is evaluated as an integral measure over the sixdimensional set of point pairs in $A$ and $B$ (see Fig.2(a)):

$$
\begin{gathered}
w_{i j k}(A, B)=\frac{1}{K_{i j k}} \int_{A} \int_{B} C_{i}\left(x_{b}-x_{a}\right) C_{j}\left(y_{b}-y_{a}\right) C_{k}\left(z_{b}-z_{a}\right) \\
d x_{a} d x_{b} d y_{a} d y_{b} d z_{a} d z_{b}
\end{gathered}
$$

where $K_{i j k}$ acts as dimensional normalization factor, and $C_{ \pm 1}($.$) are$ the characteristic functions of the positive and negative real semiaxis $(0,+\infty)$ and $(-\infty, 0)$, respectively. In particular, $C_{0}(\cdot)=$ $\delta(\cdot)$ denotes the Dirac's function, and acts as a characteristic function of the singleton set $\{0\}$. Weights between $A$ and $B$ are organized in a $3 \times 3 \times 3$ matrix $(w(A, B))$, of indexes $i, j, k$. As a particular case, it can be noticed that Eq.(1) also holds if $A$ and $B$ are coincident (i.e., $A \equiv B$ ).

In Eq.(1), the weights with one or two null indexes (i.e., $w_{i, 0,0}$, $w_{i, j, 0}, w_{i, 0, k}, w_{0, j, 0}, w_{0, j, k}, w_{0,0, k}$ and $\left.w_{0,0,0}\right)$ are computed by integrating a quasi-everywhere-null function (the set of point pairs that are aligned or coincident has a null measure in the six-dimensional space of Eq.(1)). The Dirac function appearing in the expression of $C_{0}(\cdot)$ reduces the dimensionality of the integration domain to enable a finite non-null measure. To compensate this reduction, normalization factors $K_{i, j, k}(A, B)$ have different dimensionality whether indexes $i, j$ and $k$ are equal to zero or take non-null values:

$$
\begin{array}{ll}
K_{ \pm 1, \pm 1, \pm 1}=|A||B| & K_{0,0,0}=(|A||B|)^{\frac{1}{2}} \\
K_{ \pm 1,0,0}=L_{A} L_{B} H_{A B} D_{A B} & K_{ \pm 1, \pm 1,0}=L_{A} L_{B} H_{A} H_{B} D_{A B} \\
K_{0, \pm 1,0}=L_{A B} H_{A} H_{B} D_{A B} & K_{ \pm 1,0, \pm 1}=L_{A} L_{B} H_{A B} D_{A} D_{B} \\
K_{0,0, \pm 1}=L_{A B} H_{A B} D_{A} D_{B} & K_{0, \pm 1, \pm 1}=L_{A B} H_{A} H_{B} D_{A} D_{B}
\end{array}
$$

where (see Fig.2(b)): $|A|$ and $|B|$ are the volumes of $A$, and $B ; L_{A}$, $H_{A}, D_{A}, L_{B}, H_{B}$ and $D_{B}$ are the width, height and depth of the 3D minimum embedding rectangles of $A$ and $B$, respectively; $L_{A B}$, $H_{A B}$ and $D_{A B}$ are the width, height and depth of the 3D minimum embedding rectangles of the union of $A$ and $B$, respectively.

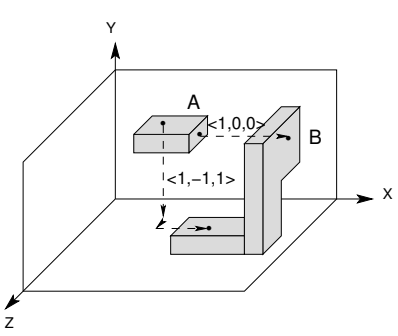

(a)

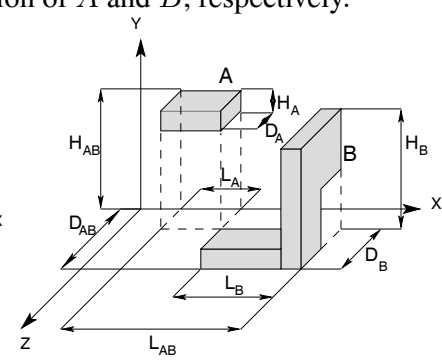

(b)
Fig. 2. (a) Walkthroughs connecting two pairs of points in entities $A$ and $B$. In each pair, the first point is in $A$, the second in $B$; (b) Measures on $A$ and $B$ appearing in normalization factors of Eq.(2).

The twenty-seven weights of the 3DWWs are reflexive (i.e., $w_{i, j, k}(A, B)=$ $\left.w_{-i,-j,-k}(B, A)\right)$, and invariant with respect to shifting and scaling.

\subsection{WWs between Linear Entities in 3D}

Computation of spatial relationships between linear entities in 3D directly descends from the general case, in that Eq.(1) sums up terms provided by individual pairs of points. In the case of 3D linear entities (curves), the main difference is that volumetric integrals become 
curvilinear integrals extended to the length of two curves. In practice, the computation is obtained by using a uniform voxelization of the space. In so doing, voxels which intersect with the curve are regarded as equivalent to 3D points and take part to the computation of the weights in Eq.(1). A trade-off between accuracy and computational complexity is used to define the grain of the hyper-rectangular decomposition.

\section{MATCHING FACE REPRESENTATIONS}

According to the modeling technique introduced in Sect.2, a generic face model $F$, is described by a set of $N_{F}$ wrinkles. In that WWs are computed for every pairs of wrinkles (including the pair composed by a wrinkle and itself), a face is represented by a set of $N_{F} \cdot\left(N_{F}+1\right) / 2$ relationship matrixes. This model is cast to a graph representation by regarding face wrinkles as graph nodes and their mutual spatial relationships as graph edges:

$$
\begin{aligned}
G \stackrel{\text { def }}{=} & <N, E, \alpha, \beta> \\
& N=\text { set of nodes } \\
& E \subseteq N \times N=\text { set of edges } \\
& \alpha: N \mapsto L_{N}, \text { nodes labeling function } \\
& \beta: E \mapsto L_{E}, \text { edge labeling function }
\end{aligned}
$$

where $L_{N}$ and $L_{E}$ are the sets of nodes and edge labels, respectively. In our framework, $\alpha$ is the function that assigns to a node $n_{k}$ the selfrelationship matrix $w\left(n_{k}, n_{k}\right)$ computed between the wrinkles associated to the node and itself. In addition, $\alpha$ associates the node with a type which distinguishes between ridge nodes and ravine nodes. The edge labeling function $\beta$ assigns to an edge $\left[n_{j}, n_{k}\right]$, connecting nodes $n_{j}$ and $n_{k}$, the relationship matrix $w\left(n_{j}, n_{k}\right)$ occurring between the wrinkles of the two nodes.

In order to compare graph representations, distance measures for node labels, and for edge labels have been defined. Both of them, rely on a distance measure $\mathcal{D}$ defined between WWs. This is derived by first computing three directional weights, taking values within 0 and 1 , on the eight corner weights of the 3DWWs matrix (all terms are intended to be computed between two curves $A$ and $B$ ):

$$
\begin{aligned}
& w_{H}=w_{1,1,1}+w_{1,-1,1}+w_{1,1,-1}+w_{1,-1,-1} \\
& w_{V}=w_{-1,1,1}+w_{1,1,1}+w_{-1,1,-1}+w_{1,1,-1} \\
& w_{D}=w_{1,1,1}+w_{1,-1,1}+w_{-1,1,1}+w_{-1,-1,1}
\end{aligned}
$$

which account for the degree by which $B$ is on the right, up and in front of $A$, respectively. Similarly, seven weights account for the alignment along the three reference directions of the space:

$$
\begin{aligned}
& w_{H_{0}}=w_{0,1,1}+w_{0,-1,1}+w_{0,1,-1}+w_{0,-1,-1} \\
& w_{V_{0}}=w_{1,0,1}+w_{-1,0,1}+w_{-1,0,-1}+w_{1,0,-1} \\
& w_{D_{0}}=w_{1,1,0}+w_{1,-1,0}+w_{-1,1,0}+w_{-1,-1,0} \\
& w_{H V_{0}}=w_{0,0,1}+w_{0,0,-1} \quad w_{H D_{0}}=w_{0,1,0}+w_{0,-1,0} \\
& w_{V D_{0}}=w_{1,0,0}+w_{-1,0,0} \quad w_{H V D_{0}}=w_{0,0,0}
\end{aligned}
$$

where $w_{H_{0}}, w_{V_{0}}, w_{D_{0}}$ measure alignments in which the coordinates $X, Y$ and $Z$ do not change, respectively; $w_{H V_{0}}, w_{H D_{0}}, w_{V D_{0}}$, measure alignments where coordinates $X Y, X Z$ and $Y Z$ do not change, respectively; and $w_{H V D_{0}}$ accounts for overlap between points of $A$ and $B$. Based on previous weights, similarity in the arrangement of pairs of curves can be evaluated in terms of a distance $D\left(w, w^{\prime}\right)$ obtained by combining the differences between homologous coefficients in the space of 27-tuples of 3DWWs. In terms of the weights of Eqs.(3)-(4), this can be expressed as:

$$
\begin{array}{r}
\mathcal{D}\left(w, w^{\prime}\right)=\lambda_{H}\left|w_{H}-w_{H}^{\prime}\right|+\lambda_{V}\left|w_{V}-w_{V}^{\prime}\right|+\lambda_{D}\left|w_{D}-w_{D}^{\prime}\right| \\
+\lambda_{H_{0}}\left|w_{H_{0}}-w_{H_{0}}^{\prime}\right|+\lambda_{V_{0}}\left|w_{V_{0}}-w_{V_{0}}^{\prime}\right|+\lambda_{D_{0}}\left|w_{D_{0}}-w_{D_{0}}^{\prime}\right| \\
+\lambda_{H V_{0}}\left|w_{H V_{0}}-w_{H V_{0}}^{\prime}\right|+\lambda_{H D_{0}}\left|w_{H D_{0}}-w_{H D_{0}}^{\prime}\right| \\
+\lambda_{V D_{0}}\left|w_{V D_{0}}-w_{V D_{0}}^{\prime}\right|+\lambda_{H V D_{0}}\left|w_{H V D_{0}}-w_{H V D_{0}}^{\prime}\right|
\end{array}
$$

where $\lambda_{H}, \lambda_{V}, \lambda_{D}, \lambda_{H_{0}}, \lambda_{V_{0}}, \lambda_{D_{0}}, \lambda_{H V_{0}}, \lambda_{H D_{0}}, \lambda_{V D_{0}}$ and $\lambda_{H V D_{0}}$, are non-negative numbers with sum equal to 1 .

Distance $\mathcal{D}$ can be proven to exhibit the five properties that are commonly assumed as axiomatic basis of metric distances. In addition, due to the integral nature of weights $w_{i j k}, \mathcal{D}$ satisfies a property of continuity which ensures that slight changes in the mutual positioning or in the distribution of points in two sets $A$ and $B$ result in slight changes in their 3DWWs. This has a main relevance in ensuring robustness of comparison.

Matching a template face graph $T$, and a gallery reference face graph $R$, involves the association of the nodes in the template with a subset of the nodes in the description. Using an additive composition, and indicating with $\Gamma$ an injective function which associates nodes $t_{k}$ in the template graph with a subset of the nodes $\Gamma\left(t_{k}\right)$ in the reference graph, this is expressed as follows:

$$
\begin{aligned}
& \mu^{\Gamma}(T, R) \stackrel{\text { def }}{=} \frac{\lambda}{N_{T}} \cdot \sum_{k=1}^{N_{T}} \mathcal{D}\left(w\left(t_{k}, t_{k}\right), w\left(\Gamma\left(t_{k}\right), \Gamma\left(t_{k}\right)\right)\right)+ \\
& +\frac{2(1-\lambda)}{N_{T}\left(N_{T}-1\right)} \cdot \sum_{k=1}^{N_{T}} \sum_{h=1}^{k-1} \mathcal{D}\left(w\left(t_{k}, t_{h}\right), w\left(\Gamma\left(t_{k}\right), \Gamma\left(t_{h}\right)\right)\right)
\end{aligned}
$$

where the first summation accounts for the average distance scored by matching nodes of the two graphs, and the second summation evaluates the mean distance in the arrangements of pairs of nodes in the two graphs. In this equation, $N_{T}$ is the number of nodes in the template graph $T$, and $\lambda \in[0,1]$ balances the mutual relevance of edge and node distance.

In general, given two graphs $T$ and $R$, a combinatorial number of different interpretations $\Gamma$ are possible, each scoring a different value of distance. According to this, the distance $\mu$ between $T$ and $R$ is defined as the minimum under any possible interpretation $\Gamma: \mu(T, R)=\min _{\Gamma} \mu^{\Gamma}(T, R)$. In so doing, computation of the distance becomes an optimal error-correcting (sub)graph isomorphism problem, which is a NP-complete problem with exponential time solution algorithms. Since the proposed modeling technique results into complete graphs with a relatively large number of nodes (i.e., typical models have more than 20 wrinkles, almost equally divided between ridges and ravines), to improve the computational efficiency, we relaxed the requirement of optimality by accepting suboptimal matches. This is obtained by imposing that cross-matches between nodes of different type is not allowed, and renouncing to include in the distance minimization the relationships between nodes of different type. According to this, the distance $\mu(T, R)$ is computed as the sum of three separated components:

$$
\begin{array}{r}
\mu(T, R)=\min _{\Gamma_{a}}\left[\mu^{\Gamma_{a}}\left(T_{a}, R_{a}\right)\right]+\min _{\Gamma_{b}}\left[\mu^{\Gamma_{b}}\left(T_{b}, R_{b}\right)\right]+ \\
+(1-\lambda) \cdot \mu_{s}\left(w\left(T_{a}, T_{b}\right), w\left(\Gamma_{a}\left(T_{a}\right), \Gamma_{b}\left(T_{b}\right)\right)\right)
\end{array}
$$

where $T_{a}, R_{a}$ and $T_{b}, R_{b}$ are the sub-graphs composed by ridge and ravine nodes in the template and reference models, respectively. Optimal solutions $\min _{\Gamma_{a}}$ and $\min _{\Gamma_{b}}$ in matching sub-graphs are computed by using the algorithm in [12]. Finally, the third term 
of Eq.(6), accounts for the relationships distance occurring between ridge nodes and ravine nodes in the matched sub-graphs:

$$
\begin{array}{r}
\mu_{s}\left(w\left(T_{a}, T_{b}\right), w\left(\Gamma_{a}\left(T_{a}\right), \Gamma_{b}\left(T_{b}\right)\right)\right)=\frac{1}{N_{T_{a}} \cdot N_{T_{b}}} . \\
\cdot \sum_{t_{k} \in T_{a}} \sum_{t_{h} \in T_{b}} \mathcal{D}\left(w\left(t_{k}, t_{h}\right), w\left(\Gamma_{a}\left(t_{k}\right), \Gamma_{b}\left(t_{h}\right)\right)\right)
\end{array}
$$

Without loss of generality, Eqs.(5)-(7) assume that the number of nodes in template graphs $\left(N_{T_{a}}, N_{T_{b}}\right)$, are not greater than the number of nodes in the reference graphs $\left(N_{R_{a}}, N_{R_{b}}\right)$. In fact, if $N_{T_{a}}>$ $N_{R_{a}}$ or $N_{T_{b}}>N_{R_{b}}$, graphs can be exchanged due to the reflexivity of 3DWWs, and the normality in the sum of their eight corner weights.

\section{EXPERIMENTAL RESULTS}

The proposed model for description and matching of faces has been experimented using models from the GavabDB database [13] which includes three-dimensional facial surface models of 61 people ( 45 male and 16 female). The whole set of people are Caucasian and most of them are aged between 18 and 40 . For each person, 7 different models are taken-differing in terms of viewpoint or facial expression-resulting in 427 facial models. In particular, there are 2 frontal and 2 rotated models with neutral facial expression, and 3 frontal models in which the person laughs, smiles or exhibits a random gesture. All models are automatically processed, as described in the previous sections, so as to extract a graph based description of their content encoding prominent characteristics of individual wrinkles as well as their relative arrangement.

In order to assess the effectiveness of the proposed solution for face identification, we performed a set of recognition experiments. In these experiments, one of the two frontal models with neutral expression provided for each person is assumed as reference (gallery) model for the identification. Results are given in Tab.1 as matching accuracy for different categories of test models.

\begin{tabular}{|l|c|}
\hline Test category & Matching Accuracy \\
\hline \hline frontal - neutral gesture & $91 \%$ \\
\hline frontal - smile gesture & $83 \%$ \\
\hline frontal - laugh gesture & $80 \%$ \\
\hline frontal - random gesture & $77 \%$ \\
\hline rotated looking down - neutral gesture & $78 \%$ \\
\hline rotated looking up - neutral gesture & $77 \%$ \\
\hline
\end{tabular}

Table 1. Matching accuracy for different categories.

It can be noted that the proposed approach provides a quite high recognition accuracy also for variations in face expression. This can be intuitively motivated by the fact that the geodesic distance between vertices of the mesh is almost invariant to facial expression changes [14]. However, large face variations as those associated to random gestures determine a lower accuracy in the recognition rate.

Finally, in Fig.3 recognition examples are reported for three test faces of different subjects. For each case, on the left the probe face is shown, while on the right the correctly identified reference face is reported. These models also provide examples of the variability in terms of facial expression of face models included in the gallery.

\section{CONCLUSIONS}

In this paper, we have proposed an original solution to the problem of $3 \mathrm{D}$ face recognition. The basic idea is to compare $3 \mathrm{D}$ face
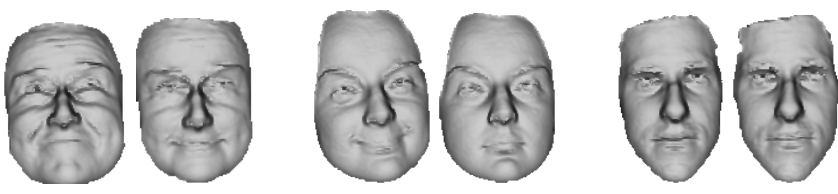

Fig. 3. Three recognition examples. For each pair, the probe (on the left) and the correctly identified model (on the right) are reported.

models by using the information provided by their salient wrinkles. To this end, an original framework has been developed which provides two main contributions. First, 3D face models are described by salient curves which are extracted as curvature ridges and ravines in $3 \mathrm{D}$ dense meshes. Then, a theory for modeling spatial relationships between curves in the 3D domain has been developed. Finally, we proposed a graph matching solution for the comparison between 3DWWs computed on curves extracted from a template model and those of reference models. The viability of the approach has been validated in a set of recognition experiments.

\section{REFERENCES}

[1] W. Zhao, R. Chellappa, P.J. Phillips, and A. Rosenfeld, "Face recognition: A literature survey," ACM Computing Survey, vol. 35, no. 4, pp. 399-458, December 2003.

[2] K.W. Bowyer, K. Chang, and P. Flynn, "A survey of approaches to three dimensional face recognition," in Proc. International Conference on Pattern Recognition, Cambridge, UK, August 2004, pp. 358-361.

[3] V. Blanz and T. Vetter, "Face recognition based on fitting a 3d morphable model," IEEE Trans. on Pattern Analysis and Machine Intelligence, vol. 25, no. 9, pp. 1063-1074, Sept. 2003.

[4] U. Park, H. Chen, and A.K. Jain, " $3 \mathrm{~d}$ model-assisted face recognition in video," in Proc. Canadian Conference on Computer and Robot Vision, May 2005, pp. 322-329.

[5] Y. Wang, C. Chua, and Y. Ho, "Facial feature detection and face recognition from 2d and 3d images," Pattern Recognition Letters, vol. 23, no. 10, pp. 1191-1202, August 2002.

[6] K. Chang, K. Bowyer, and P. Flynn, "Face recognition using 2d and 3d facial data," in Proc. Multimodal User Authentication Workshop, December 2003, pp. 25-32.

[7] A.B. Moreno, Á.Sánchez, J.F. Vélez, and F.J. Díaz, "Face recognition using 3d surface-extracted descriptor," in Proc. Irish Machine Vision and Image Precessing Conf., Sept. 2003.

[8] J. Cook, V. Chandran, S. Sridharan, and C. Fookes, "Face recognition from $3 \mathrm{~d}$ data using iterative closest point algorithm and gaussian mixture models," in Proc. Symp. on 3D Data Processing, Visualization and Transmission, Sept. 2004, pp. 502-509.

[9] V. Bruce, E. Hanna, N. Dench, P. Healey, and M. Burton, "The importance of "mass" in line drawings of faces," Applied Cognitive Psychol$o g y$, vol. 6, pp. 619-628, 1992.

[10] S. Berretti, A. Del Bimbo, and E. Vicario, "Weighted walkthroughs between extended entities for retrieval by spatial arrangement," IEEE Transactions on Multimedia, vol. 5, no. 1, pp. 52-70, March 2003.

[11] K. Watanabe and A.G. Belyaev, "Detection of salient curvature features on polygonal surfaces," Eurographics, vol. 20, no. 3, 2001.

[12] S. Berretti, A. Del Bimbo, and E. Vicario, "Efficient matching and indexing of graph models in content based retrieval," IEEE Transactions on Pattern Analysis and Machine Intelligence, vol. 23, no. 10, pp. 1089-1105, October 2001.

[13] A.B. Moreno and A. Sanchez, "Gavabdb: A 3d face database," in Proc. 2nd COST275 Workshop on Biometrics on the Internet, Vigo (Spain), March 2004, pp. 75-80.

[14] A.M. Bronstein, M.M. Bronstein, and R. Kimmel, "Three dimensional face recognition," Int. Journal of Computer Vision, pp. 5-30, 2005. 